\title{
LHC status and plans
}

\section{Mike Lamont ${ }^{* \dagger}$}

E-mail: mike.lamont@cern.ch

Following the incident in the LHC on 19 September 2008, full beam commissioning started again at the end of November 2009. An overview of the progress made since then is given. The prospects for the rest of 2010 and goals for 2011 are presented.

XVIII International Workshop on Deep-Inelastic Scattering and Related Subjects April 19 -23, 2010

Convitto della Calza, Firenze, Italy

*Speaker.

†n behalf of the LHC team. 
Table 1: LHC milestones 2009 - 2010

\begin{tabular}{ll}
\hline Date & Milestone \\
\hline 20 November & Injection of both beams, rough RF capture \\
21 November & Circulating beam 1 \\
22 November & Circulating beam 2 \\
23 November & First pilot collisions at $450 \mathrm{GeV}$ - first trial ramp \\
26 November & Pre-cycle established- energy matching \\
29 November & Ramp to $1.08 \mathrm{TeV}$ and then $1.18 \mathrm{TeV}$ \\
14 December & Ramp 2 on 2 to $1.18 \mathrm{TeV}-$ quiet beams - collisions in all four experiments \\
14 December & 16 on 16 at $450 \mathrm{GeV}-$ stable beams \\
16 December & Ramped 4 on 4 to $1.18 \mathrm{TeV}$ - collisions in all four experiments \\
\hline 27 February & First injection of 2010 \\
28 February & Circulating beams \\
5 March & Canonical two beam operation \\
8 March & Collimation setup at $450 \mathrm{GeV}$ \\
19 March & Beams to $3.5 \mathrm{TeV}$ \\
30 March & $3.5 \mathrm{TeV}$ collisions under stable beam conditions \\
23 April & Squeezed $\left(\beta^{*}=2 \mathrm{~m}\right.$.) stable beams \\
\hline
\end{tabular}

\section{Introduction}

The initial beam commissioning of the LHC in 2009 following the recovery from the 19 September 2008 incident saw remarkably rapid progress in the three and half weeks available in November to December 2009. The main commissioning goals were achieved. All key systems went through at least their initial commissioning phases. Collisions with stable beam conditions were established at $450 \mathrm{GeV}$, and the ramp to the maximum energy at the time of $1.18 \mathrm{TeV}$ was successfully attempted. Most beam-based systems became operational and LHC operations managed to start to master the control of a hugely complex system.

After a Christmas technical stop for consolidation of the quench protection system, commissioning started again in the new year. Progress was again rapid with first colliding beams at $3.5 \mathrm{TeV}$ being established under the watchful eye of the media on 30 March 2010. The main milestones from these two intense commissioning periods are summarized in table 1.

\section{Machine Status}

\subsection{Injection}

The transfer and injection process from the SPS into the LHC is complex process but operation is now well established [1].

- The transfer lines are well optimized after a rigorous measurement campaign.

- Re-phasing of the beam in the SPS, synchronization between the machines and subsequent capture worked well with only some RF controls and procedural issues as negatives. 
- Injection sequencing deals with requirements of multiple injection schemes that covered multi-bunch injection, two beams, and collision scheduling.

- The routine conditioning of the injection kickers (the so-called kicker soft start) is now part of the standard process.

- The injection quality check (IQC) process has been deployed, debugged, and become operational.

- The abort gap keeper which prevents injection of beam into the abort gap is commissioned.

A full program of beam-based checks were performed including: positioning of injection protection devices with respect to the beam, positioning of transfer line collimators, aperture checks, and kicker waveform checks [2]. A number of issues were identified, including a general issue with fast losses at injection and the BLM thresholds on shorter time-scales. Generally the performance at injection was good and clearly benefited from the experience gained during the injection tests. For the moment, however, one would worry about routinely injecting unsafe beam. It is to be noted that so-called quenchinos (resistive transitions detected by the quench protection system) were again observed with two accidental quenches caused by intensities as low as $2 \times 10^{9}$ protons.

\section{$2.2450 \mathrm{GeV}$}

A full set of instrumentation and associated hardware and software have been commissioned and made more-or-less operational. Measurement and control of the key beam parameters (orbit, tune, chromaticity, coupling, dispersion) are routine. Besides this the beam loss monitor (BLM) system performs impeccably. Beam sizes are measured using the synchrotron light monitors and wire-scanners. Lifetime optimization is performed via adjustment of tune, chromaticity, and orbit.

Energy matching between the SPS and LHC was performed and revealed only small differences between the two beams. The experiments' solenoids were brought on without fuss and the coupling and orbit perturbations corrected. LHCb and Alice's dipoles were brought on at $450 \mathrm{GeV}$ and in the ramp. There were some issues with transfer functions of these dipoles and the associated compensators which have been resolved.

Two beam operation was established both with and without separation bumps. Optics checks were performed and the beta beating measured and first attempts at correction made. A full program of polarity checks of correctors and beam position monitors was executed with only a few errors being found [3]. The availability of hardware, instrumentation and software was very impressive reflecting good preparation, very fast problem resolution and the clear benefits of leveraging $21^{\text {st }}$ century technology.

\subsection{Aperture}

A systematic set of aperture measurements was performed in the arcs and insertion regions [4]. The beam clearance in general seems to be OK, and is above or equal to expectations. Some measured bottlenecks agree with model predictions using measured beta functions. However the aperture is out of budget due to beta beating even with the closed orbit reduced to the measured 3.2 $\mathrm{mm}$ peak. This implies that correction of beta beating is mandatory at $450 \mathrm{GeV}$. 


\subsection{Optics}

The availability of measurement and impressive analysis tools should be noted. The uncorrected, measured beating was good although outside the accepted tolerance of $\approx 20 \%$ [4]. Several potential sources of error were identified with possible candidates including the warm magnets in IR3 and IR7 (large corrections required). Potential, somewhat large, corrections also pointed to the triplets in IR2 and IR8. The correction strategy will need to be carefully considered.

The pre-cycling strategy of certain classes of magnets was revisited in 2010 to avoid any potential errors arising from leaving magnets on the wrong branch of their hysteresis curves. The beating was corrected early on in the commissioning program 2010 to avoid having to re-visit collimation and other optimization after any beating corrections.

\subsection{Ramp to high energy}

A fully consistent set of machine settings was deployed at injection and for the ramp. These incorporated the output of the LHC magnet model (FIDEL [5]) which consists of all main transfer functions, dipole harmonics etc. For the RF system the necessary parameter space was in place including frequency and voltage control in the ramp.

The ramp was commissioned remarkably easily [6]. Reproducibility in the ramp looked very good enabling tune feed-forward to be deployed successfully. Tune feedback based on the continuous FFT mode of the BBQ tune system worked pretty much first time and was then used systematically during the ramp [7]. Real time acquisition of the closed orbit in the ramp was immediately available. The orbit clearly moves during the ramp but total deviations were small enough to allow good transmission. A feed-forward strategy has been established.

\subsection{Squeeze}

As of April 2010 the squeeze was commissioned to $\beta^{*}=2 \mathrm{~m}$. in all four experiments [8]. The expected factor five increase in luminosity was seen as expected with the measured $\beta^{*}$ close to expectations. The limit of $2 \mathrm{~m}$. is given by aperture (energy) and beam-beam considerations. Later in the commissioning period in fact the decision was made to back off to $3.5 \mathrm{~m}$. to ensure sufficient aperture in the experiments' insertion regions.

\subsection{Physics}

At the end of April the LHC was providing collisions with a $\beta^{*}$ of $2 \mathrm{~m}$. and 3 bunches of around $1 \times 10^{10}$ per bunch resulting in a luminosity of the order of $1 \times 10^{28} \mathrm{~cm}^{-2} \mathrm{~s}^{-1}$. Long fills with a maximum of 30 hours were delivered. Beam lifetime was excellent (over 100 hours) with these beam currents and inelastic rates typically up to $120 \mathrm{~Hz}$ when optimized with $1 \times 10^{10}$ protons per bunch. The observed luminosity lifetime was well above 20 hours. These numbers were of course rapidly surpassed with the luminosity up to $2.5 \times 10^{30} \mathrm{~cm}^{-2} \mathrm{~s}^{-1}$ by the end of July 2010 .

\section{System Commissioning}

\subsection{LHC Beam Dump System}

There was a rigorous program of measurements and tests to qualify the LHC Beam Dump System (LBDS) with beam [9]. These included: beam based alignment of the protection devices 
in the vicinity of the beam dump; aperture scans; extraction tests; asynchronous beam dump tests with de-bunched beam. Commissioning of the various sub-systems also took place: e.g. the beam energy tracking System (BETS), external post operation checks (XPOC), internal post operation checks (IPOC); interaction with the timing system, synchronization with RF and the abort gap. Inject and dump, and circulate and dump modes were successfully used operationally.

A number of issues were resolved but the performance of the LBDS was in general very good and experience thus far gives confidence in its ability to perform within its very tight specifications.

\subsection{Collimation System}

The collimation system saw excellent initial beam based commissioning following careful preparation and tests [10]. The initial phase include a full program of beam based positioning during which the hierarchy was established. Encouragingly this appeared to be respected in planned and unplanned beam loss tests there afterwards, provided the orbit had been corrected to the reference. The collimation set-up remained valid over an extended period, relying on orbit reproducibility and optics stability.

\subsection{Machine Protection System}

The machine protection system (MPS) is mission critical and will clearly be vitally important for LHC operation over the safe beam limit. In essence it comprises the beam interlock system (BIS) and the safe machine parameter system (SMP) [11]. The BIS relies on inputs from a large multitude of user. The SMP relies on services from other systems (e.g. the timing system and the bunch current transformers).

Besides this the beam drives a subtle interplay of the LBDS, the collimation system and protection devices, which rely on a well-defined aperture, orbit and optics for guaranteed safe operation. The MPS itself has worked as advertised, always pulling a beam abort when called upon to do so. There were some issues with the inputs into the SMP but the system failed safe. The LBDS, orbit, and collimation have been demonstrated as safe for the given aperture and optics. Guaranteeing this at all phases of operation remains a challenge.

\subsection{Beam Instrumentation}

In general performance was excellent. A brief summary of the performance of each system is given in table 2.

\subsection{Magnet Model}

A long and thorough magnet measurement and analysis campaign [5] meant that the deployed settings produced a machine remarkable close to the untrimmed model. In terms of tune and momentum, remarkably small discrepancies between the model and the measure machine were observed. For example, the largest momentum offsets by sector seen were: -0.27 per mil in sector 56 for beam 1 and +0.32 per mil in sector 78 for beam 2 .

The precycle was fully deployed with precyling prescriptions in place for nearly all circuits with only a handful still missing. The result was very good reproducibility. Some optimization of total length is still possible; it was taking over an hour for the full precycle. There were a number 
Table 2: Summary of LHC beam instrumentation performance

\begin{tabular}{ll}
\hline System & Performance overview \\
\hline Beam Position Monitors & $\begin{array}{l}\text { In general very good. Capture mode was commis- } \\
\text { sioned enabling multi-turn acquisition and analysis. }\end{array}$ \\
\hline Beam Loss Monitors & $\begin{array}{l}\text { Excellent performance following full deployment } \\
\text { during injection tests delivering a close to fully op- } \\
\text { erational tool. Some issues with the secondary emis- } \\
\text { sion monitors; some thresholds to be adjusted. }\end{array}$ \\
\hline Bunch Current Transformers & $\begin{array}{l}\text { Along with lifetime measurement, the systems were } \\
\text { commissioned and operational. Some calibration } \\
\text { and controls issues. }\end{array}$ \\
\hline Screens & Fully operational \\
\hline Wire scanners & $\begin{array}{l}\text { Operational, calibrated and giving reasonable num- } \\
\text { bers. }\end{array}$ \\
\hline Abort Gap Monitor & First tests were encouraging. \\
\hline Synchrotron Light Monitor & $\begin{array}{l}\text { Undulators commissioned, operational at 450 GeV } \\
\text { and 3.5 TeV. }\end{array}$ \\
\hline Tune FFT & $\begin{array}{l}\text { BBQ used routinely from day one giving tune, cou- } \\
\text { pling, and chromaticity. Used for tune feedback in } \\
\text { the ramp. Tune kickers operational }\end{array}$ \\
\hline Good progress, feedback tested, radial modulation \\
tested.
\end{tabular}

of trips of circuits during the process and it's clear that the precycle stressed the Quench Protection System (QPS) and power converters.

\subsection{Power Converters and Radio Frequency}

Superb performance of the power converters was observed with excellent tracking between reference and measured and excellent tracking between the converters around the ring.

In general, there was good performance from the key RF systems: power, beam control, low level and diagnostics [12]. Establishing capture was fast and efficient, the frequency and voltage ramps passed on the first attempts. Cogging worked well with the interaction point being repositioned to the satisfaction of the experiments. There were, however, a number of controls issues with the de-synchronization/re-synchronization process being particularly problem prone. These issues and others have been addressed. 


\section{Outlook}

\subsection{Commissioning continued}

At the end of April 2010 the remaining main objectives of LHC commissioning with beam were:

- beam commissioning continued with the main, final objective of this phase being colliding, safe, stable, squeezed beams;

- consolidation and routine 'pilot' physics at the safe beam limit of around $3 \times 10^{10}$ protons per beam at $3.5 \mathrm{TeV}$ for an extended period with machine development periods as required;

- increased intensity phase one and associated machine protection qualification - the aim is to establish secure and reproducible operation under these conditions - this phase will move the total beam intensity above the safe beam limit;

- consolidation and routine physics, again for an extended period;

- increased intensity phase two and associated machine protection qualification and so on.

\subsection{The path to higher intensities}

The destructive potential of the beams means moving very carefully at all stages. Machine protection is clearly hypercritical once the safe beam limit is passed, as is fault free operations and operational procedures. It could take some time to fully establish the latter.

The pre-requisites and detailed planning for increasing intensity are in place and will essentially cover: a full verification of aperture, orbit and optics; full verification of beam dump, protection devices, collimation, injection protection; guaranteed beam quality from injectors; a fully tested beam interlock system including transmission of safe machine parameters; fully tested hardware interlock systems; all required feedback systems operational and appropriate interlocks fully tested.

This list is not exhaustive. Resolution of all procedural, operation, controls, machine protection system, instrumentation, and hardware issues must all have been addressed. It is clear that above will not happen overnight and that a full and careful program of tests and checks is required. An extended operational running period around safe beam limit with all prerequisites in place should be pursued. This will allow confirmation that all operational procedures, controls, and instrumentation are fully functional.

After this conference the decision was made to push nominal bunch intensities into physics; this was successful and the resulting outline of the planned increase in beam intensity in 2010 is shown in table 3 .

The key issue here is the staged increase to and above $1 \mathrm{MJ}$ which is seen as as the damage threshold. An extended running period over summer at around 1.4 MJ is foreseen. This will allow thorough testing of the operations' procedures, and extended verfication of the full gamut of machine protection issues before moving on. 
Table 3: Projected intensity increase in 2010 with around nominal bunch intensity $\left(1 \times 10^{11}\right)$

\begin{tabular}{cccccccc}
\hline $\mathrm{N}_{b}$ & $\mathrm{~N}_{c}$ & $\mathrm{I}_{b}$ & $\begin{array}{c}\text { Energy per } \\
\text { beam }[\mathrm{MJ}]\end{array}$ & $\begin{array}{c}\text { Peak Luminosity } \\
{\left[\mathrm{cm}^{-2} \mathrm{~s}^{-1}\right]}\end{array}$ & $\begin{array}{c}\text { Days } \\
\text { Int. Lumi } \\
{\left[\mathrm{pb}^{-1}\right]}\end{array}$ & $\begin{array}{c}\text { Approx. } \\
\text { date }\end{array}$ \\
\hline 3 & 1 & $3 \times 10^{11}$ & 0.2 & $2.5 \times 10^{29}$ & 5 & 0.032 & W4 June \\
4 & 2 & $4 \times 10^{11}$ & 0.2 & $5.1 \times 10^{29}$ & 5 & 0.066 & W1 July \\
8 & 4 & $8 \times 10^{11}$ & 0.4 & $1.0 \times 10^{30}$ & 5 & 0.13 & W2 July \\
20 & 10 & $2 \times 10^{12}$ & 1.1 & $2.5 \times 10^{30}$ & 10 & 0.6 & W3/4 July \\
24 & 24 & $2.4 \times 10^{12}$ & 1.3 & $6.1 \times 10^{30}$ & 20 & 3.2 & August \\
48 & 48 & $4.8 \times 10^{12}$ & 2.7 & $1.2 \times 10^{31}$ & 10 & 3.1 & September \\
96 & 96 & $9.6 \times 10^{12}$ & 5.4 & $2.4 \times 10^{31}$ & 10 & 6.2 & September \\
144 & 144 & $1.4 \times 10^{13}$ & 8.1 & $3.6 \times 10^{31}$ & 10 & 9.3 & October \\
192 & 192 & $1.9 \times 10^{13}$ & 10.8 & $4.9 \times 10^{31}$ & 10 & 12.7 & October \\
240 & 240 & $2.4 \times 10^{13}$ & 13.4 & $6.1 \times 10^{31}$ & 7 & 11.1 & November \\
\hline
\end{tabular}

\section{The near future}

- Proton physics in 2010 will continue at $3.5 \mathrm{TeV}$ with a target integrated luminosity target of the order of $100 \mathrm{pb}^{-1}$.

- The schedule for 2010 also includes a 5 week lead ion run.

- There will be a short winter technical stop 2010-2011 of around 2 months.

- 2011 will see an extended run at $3.5 \mathrm{TeV}$ with a target luminosity greater than $10^{32} \mathrm{~cm}^{-2} \mathrm{~s}^{-1}$ with the aim of delivering at least $1 \mathrm{fb}^{-1}$ by the end of the year [13].

- A long shutdown is planned for 2010 to fully consolidate the splices in the interconnects. This will open the way for the LHC to move safely to $7 \mathrm{TeV}$.

\section{Conclusions}

A lot of hard work over the years has enable a truly impressive period of commissioning with beam. Initial indications are that the LHC: is reproducible; magnetically well understood; optically in good shape; is armed with a excellent set of instrumentation, software, and hardware systems. There is still lots to sort out, in particular operations, controls, instrumentation etc. still have the capability to unnecessarily stress the machine protection system and there are a number of important issues to be resolved. It is clear that there is a long way to go before we are ready to move too far above the safe beam limit.

The first collisions at $3.5 \mathrm{TeV}$ squeezed were delivered towards the end of April 2010 at the time of this conference. This was followed by a running period around the safe beam limit and rigorous preparation for moving beyond the safe beam limit. Thereafter a policy of careful stepwise increase in intensity is to be pursued. Each each step up in intensity will be followed by an extended running period . 
The targets for the next two years are clearly defined with $1 \mathrm{fb}^{-1}$ being the integrated luminosity target for the end of 2011.

First indications are that the LHC is a beautiful machine and thanks are due to the many teams involved. A remarkable effort has been repaid with remarkably well behaved machine and excellent progress so far.

\section{References}

[1] M. Meddahi, LHC Injection and Transfer lines, Proceedings of the 2010 Evian workshop on LHC commissioning 19-20th January 2010, CERN-ATS-2010-028.

[2] W. Bartmann, Injection and dump protection, Proceedings of the 2010 Evian workshop on LHC commissioning 19-20th January 2010, CERN-ATS-2010-028.

[3] K. Fuchsberger,Orbit system including feedback and stability, Proceedings of the 2010 Evian workshop on LHC commissioning 19-20th January 2010, CERN-ATS-2010-028.

[4] R. Thomas, LHC optical model and necessary corrections, Proceedings of the 2010 Evian workshop on LHC commissioning 19-20th January 2010, CERN-ATS-2010-028.

[5] E. Todesco, Updated magnetic model for ramp and squeeze, Proceedings of the 2010 Evian workshop on LHC commissioning 19-20th January 2010, CERN-ATS-2010-02.

[6] W. Venturini, Ramp: experience and issues, Proceedings of the 2010 Evian workshop on LHC commissioning 19-20th January 2010, CERN-ATS-2010-028.

[7] R. Steinhagen, M. Gasior, Tune, chromaticity, feed-forward and feedback, Proceedings of the 2010 Evian workshop on LHC commissioning 19-20th January 2010, CERN-ATS-2010-028.

[8] S. Redaelli, Squeeze: strategy and issues, Proceedings of the 2010 Evian workshop on LHC commissioning 19-20th January 2010, CERN-ATS-2010-028.

[9] J. Uythoven, Beam Dump Systems and Abort Gap Cleaning, Proceedings of the 2010 Evian workshop on LHC commissioning 19-20th January 2010, CERN-ATS-2010-028.

[10] C. Bracco, Collimators and beam cleaning: first results and future plans, Proceedings of the 2010 Evian workshop on LHC commissioning 19-20th January 2010, CERN-ATS-2010-028.

[11] B. Todd, BIS, BIC and SMP, Proceedings of the 2010 Evian workshop on LHC commissioning 19-20th January 2010, CERN-ATS-2010-028.

[12] A. Butterworth, RF - performance and operational issues, Proceedings of the 2010 Evian workshop on LHC commissioning 19-20th January 2010, CERN-ATS-2010-028.

[13] M. Lamont, Luminosity estimates, Proceedings of the Chamonix 2010 Workshop on LHC Performance, CERN-ATS-2010-026. 